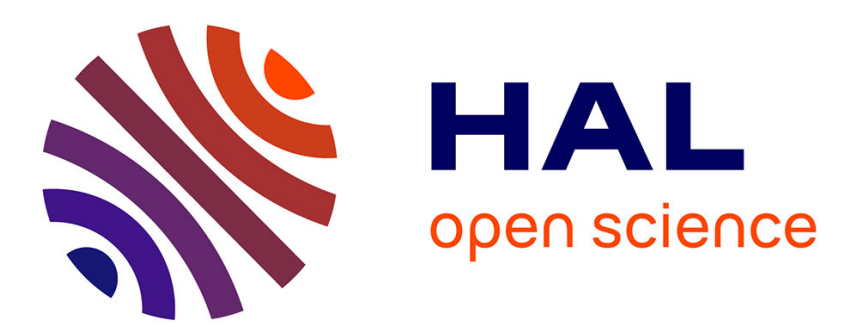

\title{
Transfert inductif d'énergie à haut rendement par procédé non dissipatif
}

\author{
A. Delmas
}

\section{To cite this version:}

A. Delmas. Transfert inductif d'énergie à haut rendement par procédé non dissipatif. Revue de Physique Appliquée, 1989, 24 (11), pp.1029-1038. 10.1051/rphysap:0198900240110102900 . jpa00246139

\section{HAL Id: jpa-00246139 https://hal.science/jpa-00246139}

Submitted on 1 Jan 1989

HAL is a multi-disciplinary open access archive for the deposit and dissemination of scientific research documents, whether they are published or not. The documents may come from teaching and research institutions in France or abroad, or from public or private research centers.
L'archive ouverte pluridisciplinaire HAL, est destinée au dépôt et à la diffusion de documents scientifiques de niveau recherche, publiés ou non, émanant des établissements d'enseignement et de recherche français ou étrangers, des laboratoires publics ou privés. 
Classification

Physics Abstracts

84.60Td

\title{
Transfert inductif d'énergie à haut rendement par procédé non dissipatif
}

\author{
A. Delmas \\ Laboratoire d'Electrotechnique des Universités Paris VI et XI (UA 845), Bât. 214, 91405 Orsay Cedex, France
}

(Reçu le 19 janvier 1989, révisé le 26 juin 1989, accepté le 5 septembre 1989)

\begin{abstract}
Résumé. - Le transfert rapide de hautes énergies entre deux inductances s'avère indispensable dans de nombreuses applications telles la propulsion électromagnétique, la réalisation de sources impulsionnelles de forte énergie, etc... Cette opération se heurte au problème crucial du rendement. Il est toutefois possible de contourner cet obstacle en réalisant, à l'aide d'un jeu de commutations, une désactivation progressive des spires d'une des inductances. Nous développons ici, l'étude de ce concept de transfert inductif séquentiel d'énergie à haut rendement sur un système, permettant, grâce à l'assistance de capacités de faible énergie et d'un transformateur de faible puissance, l'accès à une source d'énergie modulable et directement intégrable aux sources impulsionnelles du type génératrice sans fer développé par ailleurs.
\end{abstract}

Abstract. - This paper deals with fast high energy transfer between two coils in order to make a pulsed energy supply or an electromagnetic launching device. A new high efficiency system is studied. The commutation energy at each elementary step of the transfer is transiently stored in a low energy capacitor which is coupled to different entry points of the storage coil with a transformer.

\section{Introduction.}

De nombreux travaux, en électrotechnique impulsionnelle de grande puissance, se heurtent au problème de déplacement rapide et à haut rendement, de fortes énergies entre deux inductances (sources électromécaniques impulsionnelles [9], propulseurs électromagnétiques, magnéto-formage, etc...).

A un autre niveau de puissance, nous rencontrons, dans le domaine plus classique de la distribution et de la conversion de puissance, des problèmes similaires sous la forme d'indispensables brusques inversions de courants dans des circuits inductifs (type collecteurs) ou de leur simple annulation (disjonction) pouvant conduire à une assistance électronique ou électromagnétique.

Ce problème général de modification rapide d'une distribution locale d'énergie magnétique dans les circuits de puissance a déjà été abordé et aboutit voilà une dizaine d'années à la formulation, dans un contexte impulsionnel, du concept de transfert inductif d'énergie à haut rendement $[1,2]$.

Ce concept a été repris d'ailleurs plus récemment outre Atlantique par Zucker [3, 4].
Principe.

Le transfert inductif d'énergie s'appuie sur quelques principes simples [1] que nous allons rappeler.

Le simple transfert d'énergie d'une inductance pure $L_{1}$ dans une autre inductance pure $L_{2}$ (non couplée à la précédente), peut être assuré par l'ouverture d'un interrupteur commun aux deux bobines (Fig. 1). L'évolution énergétique du système peut être déduite de la loi de conservation du flux d'induction magnétique total. Elle peut être représentée dans un repère portant en abscisse et en ordonnée respectivement les racines carrées des énergies magnétiques dans $L_{1}$ et $L_{2}$. Le rendement de l'opération est alors déplorable (sa valeur maximale de $25 \%$ est obtenue pour $L_{1}=L_{2}$ ).

Pour contourner cette difficulté on peut d'une part conférer un fort taux de couplage aux spires de la bobine de stockage et d'autre part envisager un transfert séquentiel. Nous sommes alors conduit, par un jeu de commutations adaptées, à désactiver progressivement les spires de la bobine de stockage. La figure 2 visualise une séquence de transfert élémentaire. L'évolution énergétique du système est 


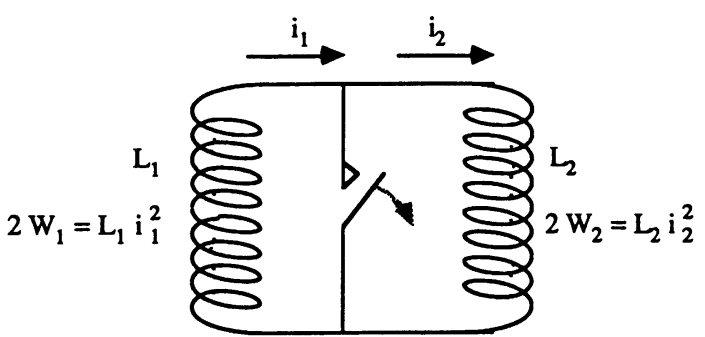

$$
\begin{array}{lll}
\text { Conditions initiales: } & t=0 & i_{1}=i_{10} ; i_{2}=0 \\
\text { et finales: } & t=\infty & i_{1}=i_{2}=I
\end{array}
$$

$$
L_{1} i_{1}+L_{2} i_{2}=c t e=L_{1} i_{10}=\left(L_{1}+L_{2}\right) I
$$

Fig. 1. - Transfert d'énergie entre deux inductances.

[Energy transfer between two coils.]

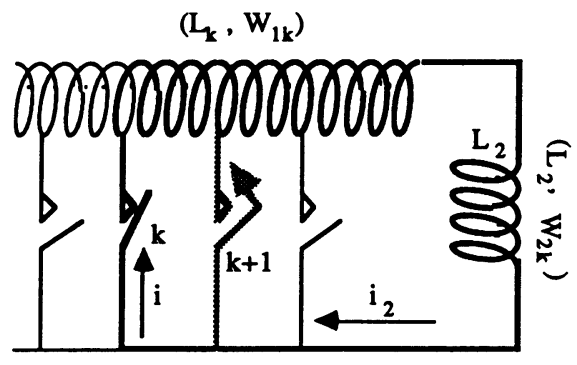

Phase 1

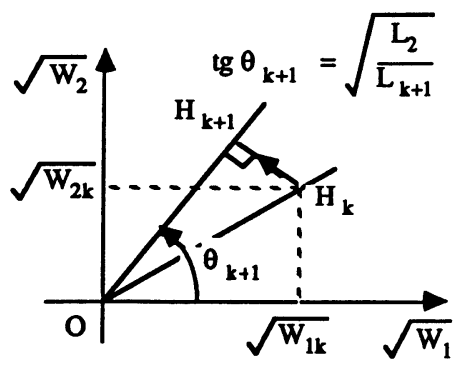

Evolution énergétique du système

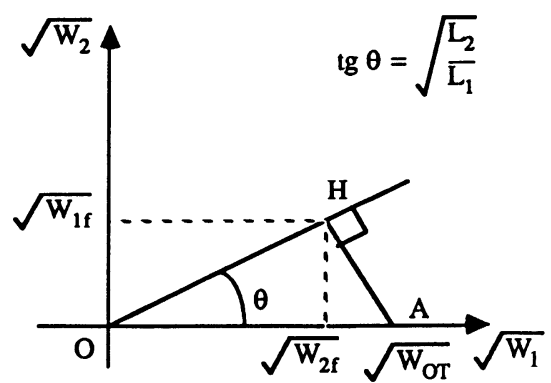

$$
\begin{gathered}
\sqrt{\mathrm{L}_{1}} \sqrt{\mathrm{W}_{1}}+\sqrt{\mathrm{L}_{2}} \sqrt{\mathrm{W}_{2}}=\mathrm{cte}=\sqrt{\mathrm{L}_{1}} \sqrt{\mathrm{W}_{\mathrm{OT}}} \\
\sqrt{\frac{\mathrm{W}_{2 \mathrm{f}}}{\mathrm{W}_{1 \mathrm{f}}}}=\sqrt{\frac{\mathrm{L}_{2}}{\mathrm{~L}_{1}}}
\end{gathered}
$$

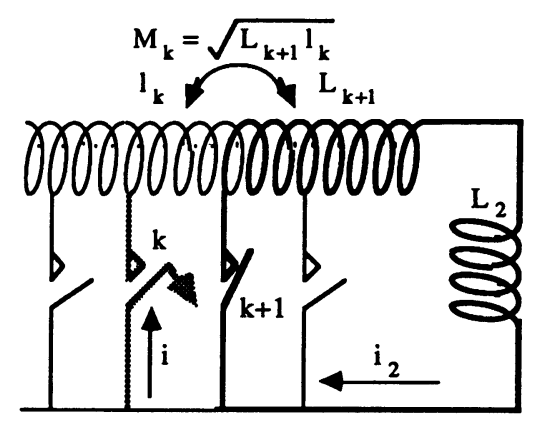

Phase 2

\section{Loi de conservation du flux}

$\left(L_{2}+L_{k+1}\right) i_{2}+\sqrt{1_{k} L_{k+1}} i=c t e$

Fig. 2. - Transfert inductif d'énergie séquentiel.

[Sequential inductive energy transfer.]

ici plus satisfaisante. Il est possible de minimiser l'énergie dissipée durant les commutations (Fig. 2) et d'atteindre de bons rendements à partir d'un nombre modéré de séquences (plus de 0,7 au-delà de 6 séquences) [1].

Toutefois de sérieux problèmes apparaissent au niveau des interrupteurs: nous devons associer l'absorption rapide de l'énergie de commutation à un recouvrement diélectrique satisfaisant. (Les fusibles sont sur le premier point fort performants, mais défaillants sur le deuxième $[5,6])$. A ces difficultés s'ajoutent celles liées à la réalisation de bobines de stockage à taux de couplage inter-spires très élevés.

Pour éliminer ces inconvénients, nous avons développé l'idée, déjà formulée il y a quelques années par Rioux [1, 7], du stockage transitoire de l'énergie de commutation dans une capacité (Fig. 3). Nous avons ainsi accès à un excellent rendement et, de plus, la possibilité d'ouvrir les interrupteurs après extinction naturelle du courant de conduction. 


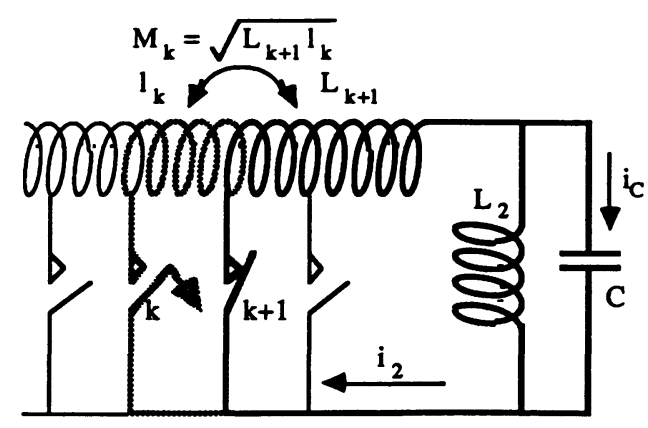

a) Mode dissipatif

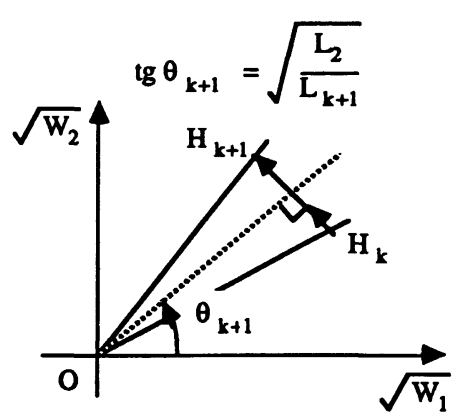

b) Mode non dissipatif

Fig. 3. - Transfert inductif d'énergie séquentiel assisté par un condensateur.

[Sequential inductive energy transfer assisted by a capacitor.]

Transfert non dissipatif assisté par un transformateur.

La configuration initiale fait appel à un condensateur placé directement aux bornes de l'inductance d'utilisation (Fig. 3). Dès ouverture de l'interrupteur $k(k+1$ étant fermé) le courant qui le traverse s'annule. Simultanément nous avons un brusque accroissement du courant dans la capacité (durant ce laps de temps nous avons conservation du flux d'induction magnétique dans l'inductance de stockage). Au cours de l'oscillation qui suit elle assure un stockage transitoire d'énergie (de commutation) qu'elle restitue ensuite aux inductances en service. Dès cet instant on assure la fermeture de $k+2$ et ensuite l'ouverture de $k+1$.

Cette solution présente au niveau pratique quelques sérieux inconvénients. Les inévitables défauts de couplage au niveau de la bobine de stockage sont toujours sources de pertes d'énergie au niveau des interrupteurs. De plus, dans ce contexte, nous avons à commuter de plus en plus rapidement des courants de plus en plus intenses (du fait de la diminution de l'inductance de la bobine de stockage). Cela conduit à des contraintes trop sévères pour les interrupteurs. Tout autre position de la capacité présente aussi son lot équivalent de désagréments.

Afin d'apporter une solution à ces problèmes parasites d'absorption d'énergie, d'adaptation de la capacité à chaque séquence, et accéder à un transfert d'énergie modulable, nous faisons appel à un transformateur (Fig. 4).

Isolons une séquence de transfert $k$ où les interrupteurs $k, k+1$, sont fermés. Supposons qu'elle soit caractérisée à l'instant initial $(t=0)$ par :

$$
\begin{gathered}
V_{C k}(t=0)=0 ; \quad i_{k}(t=0)=I_{k}=j_{k}(t=0) ; \\
j_{k+1}(t=0)=0 .
\end{gathered}
$$

Nous avons, durant l'oscillation du système ( $V_{C k}$ devient positif), et si les conditions énergéti-

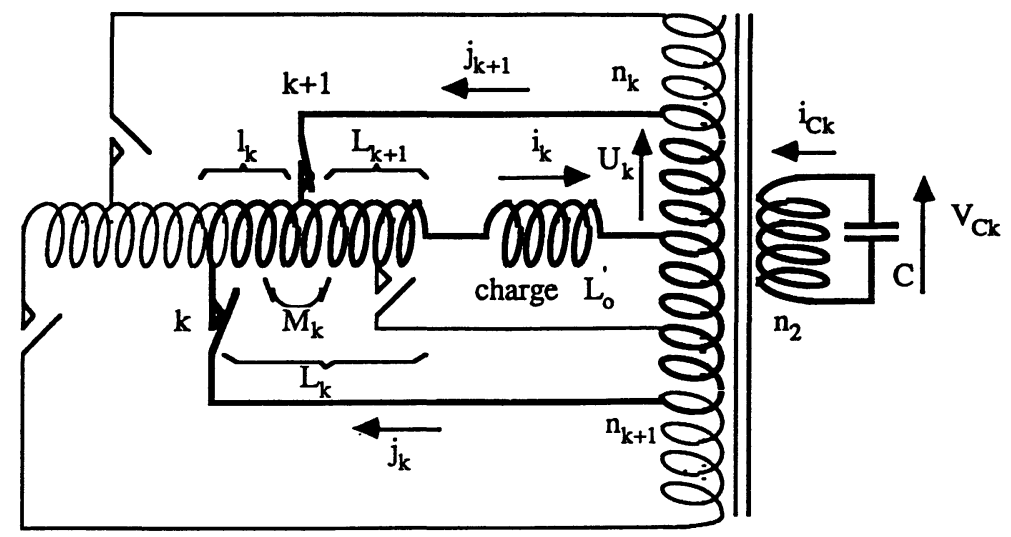

Fig. 4. - Transfert inductif d'énergie séquentiel assisté par un condensateur variable.

[A non dissipative transfer system.] 
ques sont favorables, annulation du courant dans la branche dotée de l'interrupteur $k$. Il est alors aisé d'ouvrir cette portion du circuit (par exemple par extinction naturelle d'un arc électrique sous vide).

\section{Conditions de fonctionnement et optimisation.}

La formulation des conditions de bon fonctionnement du système s'appuie sur la constante du flux d'induction magnétique dans les inductances $\ell_{k}$, $L_{k+1}$ (couplées) et $L_{0}^{\prime}$ lors de la $k$-ième séquence :

$$
\begin{aligned}
& {\left[\left(1+\frac{n_{k}}{n_{k+1}}\right)\left(L_{k+1}+L_{0}^{\prime}\right)+M_{k}\right] i_{k}+} \\
& +\left[\left(1+\frac{n_{k}}{n_{k+1}}\right) M_{k}+\ell_{k}\right] j_{k}=\text { Cte }
\end{aligned}
$$

avec $n_{k}$ et $n_{k+1}$ les nombres de spires du bobinage primaire du transformateur entre $L_{0}^{\prime}$ et respectivement les interrupteurs $k, k+1$ (Fig. 4). On peut aussi obtenir la relation ci-dessus à partir des équations (5) et (6).

Nous avons alors entre les deux courants de début $\left(I_{k}\right)$ et de fin $\left(I_{k+1}^{\prime}\right)$ du transfert élémentaire, les relations :

$$
\begin{aligned}
& \frac{I_{k}}{I_{k+1}^{\prime}}= \\
& \quad=\frac{L_{k+1}+L_{0}^{\prime}+M_{k}+\left(1+\frac{n_{k}}{n_{k+1}}\right)\left(L_{k+1}+L_{0}^{\prime}\right)}{L_{k}+L_{0}^{\prime}+\frac{n_{k}}{n_{k+1}}\left(L_{k+1}+L_{0}^{\prime}+M_{k}\right)} .
\end{aligned}
$$

Nous avons apparition ici de $L_{k}$, résultat de l'association des inductances $L_{k+1}$ et $\ell_{k}$ via celle de couplage $M_{k}$ :

$$
L_{k}=L_{k+1}+\ell_{k}+2 M_{k} .
$$

Nous devons avoir également annulation du courant dans $k$ durant la première demi-période $\left(T_{k} / 2\right)$, c'est-à-dire avant retour à zéro de $V_{C k}$ (ou plus exactement de $\left.V_{C k}\left(t=T_{k} / 2\right)\right)$. Ceci nous conduit à l'inégalité :

$$
\frac{1}{2}\left(L_{0}^{\prime}+L_{k}\right) I_{k}^{2} \geqslant \frac{1}{2}\left(L_{0}^{\prime}+L_{k+1}\right) I_{k+1}^{\prime 2}
$$

L'association des deux relations (1) et (2) nous donne finalement :

$$
\frac{n_{k}}{n_{k+1}} \geqslant \sqrt{\frac{L_{0}^{\prime}+L_{k}}{L_{0}^{\prime}+L_{k+1}}} .
$$

L'optimisation du fonctionnement du système est obtenue pour un passage du courant $j_{k}$ à 0 lorsque $V_{C k}$ devient nul. Il est décrit par la double égalité :

$$
\frac{I_{k+1}}{I_{k}}=\frac{n_{k}}{n_{k+1}}=\frac{\sqrt{L_{0}^{\prime}+L_{k}}}{\sqrt{L_{0}^{\prime}+L_{k+1}}}
$$

où $I_{k+1}$ est la valeur optimale de $I_{k+1}^{\prime}$.

$\mathrm{Si}$, de plus, au cours du transfert nous imposons que la charge qui transite par la capacité soit identique pour chacune des séquences nous aurons alors la période d'oscillation du système qui restera constante $\left(T_{k}=T_{0}\right)$. Nous avons en effet ici :

$$
C\left[V_{C k}\right]_{\mathrm{crête}}=\mathrm{Cte}=\frac{T_{k}}{2 \pi} \frac{n_{k} I_{k}}{n_{2}} .
$$

Dans ces conditions de fonctionnement bien définies nous pouvons prédéterminer entièrement l'évolution électrique du système.

\section{Equations d'évolution électriques.}

Les équations d'évolution temporelles des courants et tensions dans les différentes parties du circuit sont d'écriture aisée (cf. Fig. 4). Nous avons :

- pour les tensions :

$$
\begin{aligned}
& \frac{n_{k+1}}{n_{2}} V_{C k}=U_{k}=\left(L_{k+1}+L_{0}^{\prime}\right) \frac{\mathrm{d} i_{k}}{\mathrm{~d} t}+M_{k} \frac{\mathrm{d} j_{k}}{\mathrm{~d} t} \\
& \qquad\left(1+\frac{n_{k}}{n_{k+1}}\right) U_{k}=-\ell_{k} \frac{\mathrm{d} j_{k}}{\mathrm{~d} t}-M_{k} \frac{\mathrm{d} i_{k}}{\mathrm{~d} t} \\
& \text { - et pour les courants : } \\
& n_{k+1} j_{k+1}-n_{k} j_{k}=n_{2} i_{C k}=-n_{2} C \frac{\mathrm{d} V_{C k}}{\mathrm{~d} t} \\
& j_{k+1}+j_{k}=i_{k} .
\end{aligned}
$$

Pour tenir compte des caractéristiques réelles des interrupteurs (tension négative après extinction du courant), on est amené à modifier les conditions initiales précédemment écrites et à prendre :

$$
V_{C k}=-\varepsilon_{k} ; \quad i_{k}=I_{k}=j_{k} ; \quad j_{k+1}=0 .
$$

Il nous paraît raisonnable de ne faire intervenir les pertes qu'après cette première approche, à l'aide d'un correctif adapté à la configuration de la bobine de stockage et des éléments de commutation retenus. Celles-ci demeurent a priori réduites.

De ces équations nous déduisons l'expression de la période d'oscillation :

$$
\begin{aligned}
&\left(\frac{T_{k}}{2 \pi}\right)^{2}=\left(\frac{n_{2}}{n_{k+1}}\right)^{2} C \times\left(\ell_{k}\left(L_{k+1}+L_{0}^{\prime}\right)-M_{k}^{2}\right) \\
& \times \frac{n_{k}}{\ell_{k}+2\left(1+\frac{n_{k}}{n_{k+1}}\right)^{2} \times} \times\left(1+\frac{\left(M_{k+1}\right.}{n_{k}} \times\left(L_{0}^{\prime}+L_{k+1}\right)\right.
\end{aligned}
$$


Nous avons donc :

$$
V_{C k}=V_{C_{0} k} \sin \left(\frac{2 \pi}{T_{k}} t-\varphi_{k}\right)
$$

avec

$$
V_{C_{0} k} \sin \varphi_{k}=\varepsilon_{k} \text {. }
$$

Il ne reste plus qu'à optimiser le fonctionnement du système tant au niveau de chaque séquence que de leur juxtaposition :

$$
\begin{aligned}
V_{C_{0} k}=V_{C_{0}} ; \quad \varepsilon_{k} & =\varepsilon_{0} ; \quad \varphi_{k}=\varphi \\
T_{k}=T_{0} ; \quad \frac{n_{k}}{n_{k+1}} & =\sqrt{\frac{L_{k}+L_{0}^{\prime}}{L_{k+1}+L_{0}^{\prime}}} .
\end{aligned}
$$

\section{Caractéristiques électriques optimales.}

Nous cherchons à définir ici les inductances mises en jeu au cours des séquences, et à préciser ainsi, pour une bobine de stockage donnée, la matrice inductance de transfert idéale. Ces séquences, optimisées, mettent en jeu des grandeurs invariantes, telles la période d'oscillation $T_{0}$ du système, la charge véhiculée $C V_{C_{0}}$, les conditions d'amorçage des séquences $\left(\varphi_{k}=\varphi\right)$. Nous avons :

$$
C V_{C_{0}}=\frac{T_{0} i_{C_{0}}}{2 \pi \cos \varphi}=\frac{T_{0} I_{k}}{2 \pi \cos \varphi} \frac{n_{k}}{n_{2}}
$$

$i_{C_{0}}$ : courant capacitif crête

$n_{2}:$ nombre de spires au secondaire du transformateur.

En faisant intervenir l'énergie de commutation, fraction $\alpha^{\prime}$ de l'énergie magnétique initiale $W_{0 T}$ mise en jeu, et la liaison de la période $T_{0}$ aux caractéristiques électriques du circuit (les inductances $\ell_{k}, L_{k+1}$ et de leur mutuelle $M_{k}$ ) nous obtenons :

$$
\begin{array}{r}
\alpha^{\prime}=\alpha \cos ^{2} \varphi= \\
=\frac{\ell_{k}\left(L_{k+1}+L_{0}^{\prime}\right)-M_{k}^{2}}{\left(L_{k+1}+L_{0}^{\prime}\right)\left\{\ell_{k}+2\left(1+\frac{n_{k}}{n_{k+1}}\right) M_{k}+\right.} \\
\left.+\left(1+\frac{n_{k}}{n_{k+1}}\right)^{2}\left(L_{k+1}+L_{0}^{\prime}\right)\right\}
\end{array}
$$

avec

$$
\begin{aligned}
\frac{n_{k}}{n_{k+1}} & =\sqrt{\frac{L_{0}^{\prime}+L_{k}}{L_{0}^{\prime}+L_{k+1}}} \\
\text { et } \quad \frac{L_{k}}{L_{k+1}} & =1+\frac{\ell_{k}}{L_{k+1}}+\frac{2 M_{k}}{L_{k+1}} .
\end{aligned}
$$

$\alpha$ représente le taux d'énergie totale qui transite par la capacité, dont une fraction $\alpha^{\prime}$ sert à la commutation proprement dite alors que la part restante $\left(\alpha-\alpha^{\prime}\right)$ est utilisée pour le blocage postconduction de l'interrupteur.

L'inductance $L_{k+1}$ étant connue, il est possible de déduire $\ell_{k}$ ou $L_{k}$ en fonction de $\alpha^{\prime}$. Cette opération peut être reproduite $N$ fois entre deux positions limites $\left(L_{0}, L_{N}\right)$. Un ajustement de la valeur de $\alpha^{\prime}$, va nous permettre de dégager finalement $N$ séquences énergétiquement identiques. Nous obtenons ainsi, outre la valeur du taux d'énergie de commutation $\alpha^{\prime}$, celle des éléments $\left(\ell_{k}, L_{k}, M_{k}\right)$ de la matrice inductance d'une bobine de stockage donnée.

Nous pouvons réécrire les relations précédentes en faisant apparaître des termes sans dimensions. Pour ce nous faisons appel à une représentation angulaire de l'évolution énergétique du système en posant :

$\operatorname{tg} \Phi_{k+1}=\sqrt{\frac{L_{0}^{\prime}}{L_{k+1}}}$ avec $\frac{\pi}{2}>\Phi_{k+1}>\Phi_{0}>0$

$$
M_{k}=C_{k} \sqrt{\ell_{k} L_{k+1}} .
$$

Nous obtenons :

$$
\begin{aligned}
\sqrt{\frac{\ell_{k}}{L_{k+1}}} & =\sqrt{\frac{\ell_{k}}{L_{0}^{\prime}}} \operatorname{tg} \Phi_{k+1} \\
& =\frac{2\left(X_{k}+C_{k} \cos ^{2} \Phi_{k+1}\right)}{X_{k}^{2}-\cos ^{2} \Phi_{k+1}}
\end{aligned}
$$

avec

$X_{k}=\cos ^{2} \Phi_{k+1} \times$

$$
\times\left\{-C_{k}+\sqrt{\left(\frac{1}{\cos ^{2} \Phi_{k+1}}-C_{k}^{2}\right)\left(\frac{1}{\alpha^{\prime}}-1\right)}\right\}
$$$$
\frac{L_{k}}{L_{k+1}}=\left(\sqrt{\frac{\ell_{k}}{L_{k+1}}+C_{k}}\right)^{2}+1-C_{k}^{2}=\frac{\operatorname{tg}^{2} \Phi_{k+1}}{\operatorname{tg}^{2} \Phi_{k}} \text {. }
$$

- Couplage parfait $\left(M_{k}=\sqrt{\ell_{k} L_{k+1}}\right)$.

Cette situation idéale va avoir une formulation simple. En prenant comme référence l'évolution énergétique angulaire telle qu'indiquée sur la figure 3 nous obtenons :

$$
\sin ^{2} \frac{\Phi_{k+1}-\Phi_{k}}{2}=\alpha^{\prime}=\alpha \cos ^{2} \varphi .
$$

La prédétermination de l'évolution temporelle des courants et tensions dans les différentes parties du circuit est alors aisée, pour peu que l'on connaisse le nombre de séquences $N$ et les positions énergétiques initiale $\left(\Phi_{0}\right)$ et finale $\left(\Phi_{N}\right)$ de l'opération de transfert (Fig. 5). Il peut être utile de faire apparaître 


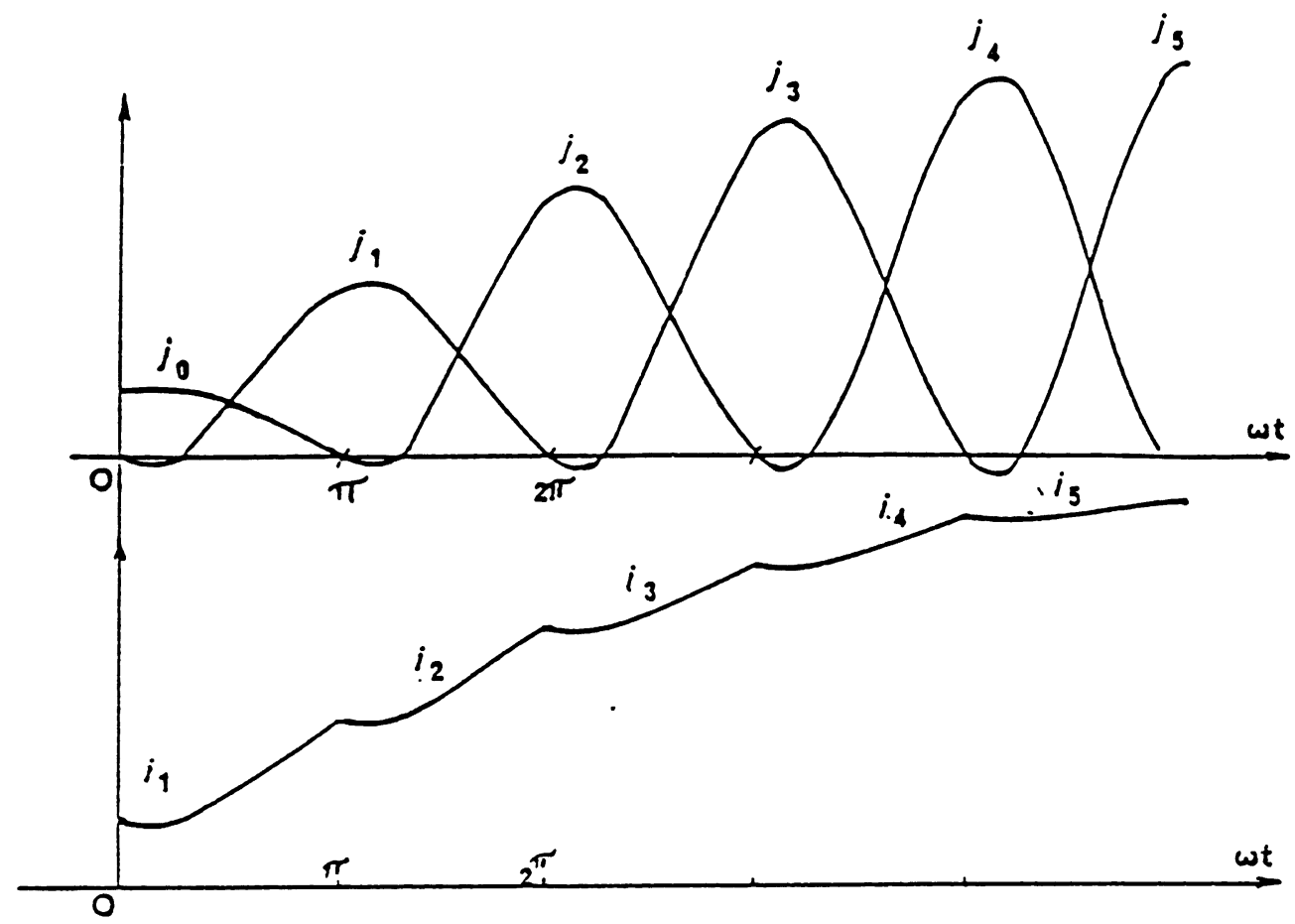

a)

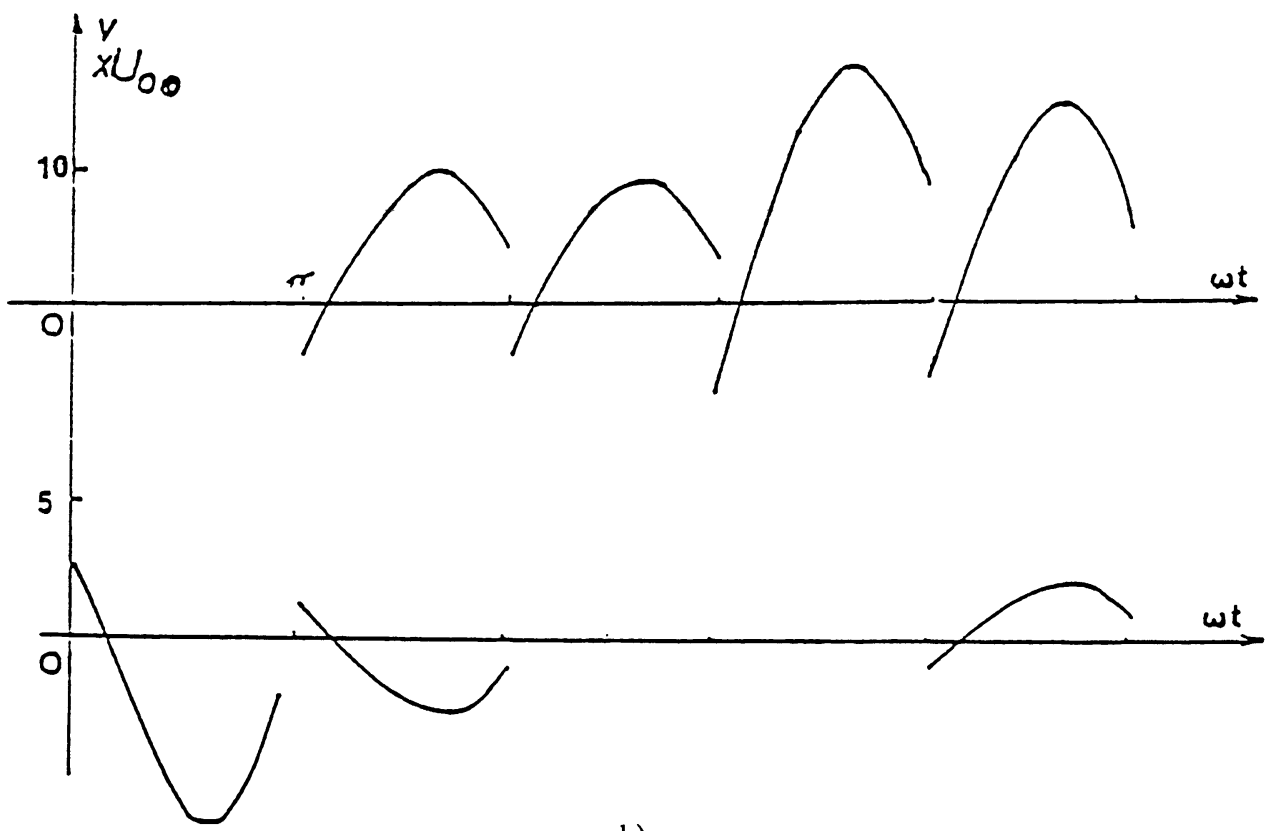

b)

Fig. 5. - Diagramme synthétique des courants a) et tensions b) $\left(\Phi_{0}=\pi / 18, \Phi_{N}=\pi / 2, N=5, \varphi=0,133 \pi\right.$, $\beta=5,56, \alpha^{\prime}=1,94 \times 10^{-2}$ ). a) Courant dans les interrupteurs et la charge. b) Tension dans les interrupteurs 1 et 3 .

[a) Current waveform. b) Voltage waveform.]

ici le gain en courant $\beta$, rapport du courant final $I_{N}$ au courant initial $I_{0}$. Il aura pour expression :

$$
\beta=\frac{I_{N}}{I_{0}}=\frac{\sin \Phi_{N}}{\sin \Phi_{0}} .
$$

Pour s'approcher d'une efficacité de transfert d'énergie maximale, il paraît raisonnable d'arrêter le transfert lorsqu'il y a égalité entre les énergies transférées au cours de la première et de la dernière séquence. On a alors :

$$
\Phi_{N}=\frac{\pi}{2}-\Phi_{0} .
$$




\section{- Couplage nul $\left(M_{k}=0\right)$.}

Cette situation perd tout sens dans le cadre du transfert d'énergie réalisé selon le procédé dissipatif (absence de capacité), mais ici, elle apparaît tout au moins dans son principe, possible.

Dans la représentation angulaire de l'évolution énergétique du système nous avons selon les mêmes repères, la relation :

$$
\frac{\sin \Phi_{k}}{\sin \Phi_{k+1}}=1-2 \alpha^{\prime}
$$

Il est clair qu'ici, les dernières séquences sont, sur le plan transfert d'énergie, les plus performantes. Par contre les taux d'énergie de commutation impliqués sont nécessairement plus importants que précédemment.

\section{Flux d'induction magnétique admissible.}

Le flux d'induction magnétique total impliqué au cours du transfert d'énergie est une grandeur caractéristique liée à la durée des séquences et à la tension crête apparaissant aux bornes de la capacité.

Lors de la séquence $k$, ce flux $\xi_{k}$ lié à l'énergie magnétique initiale $W_{0 T}$, pourra, compte tenu de (10), s'exprimer par la relation :

$$
\begin{aligned}
\xi_{k}=\sqrt{2 W_{0 T}} & \sqrt{L_{0}^{\prime}+L_{k}}= \\
= & \frac{T_{0} V_{C_{0}}}{2 \pi \alpha \cos \varphi} \frac{n_{k}}{n_{2}}=\frac{n_{k}}{n_{k+1}} \xi_{k+1} \\
& \left(2 \alpha W_{0} T=C V_{C_{0}}^{2}\right) .
\end{aligned}
$$

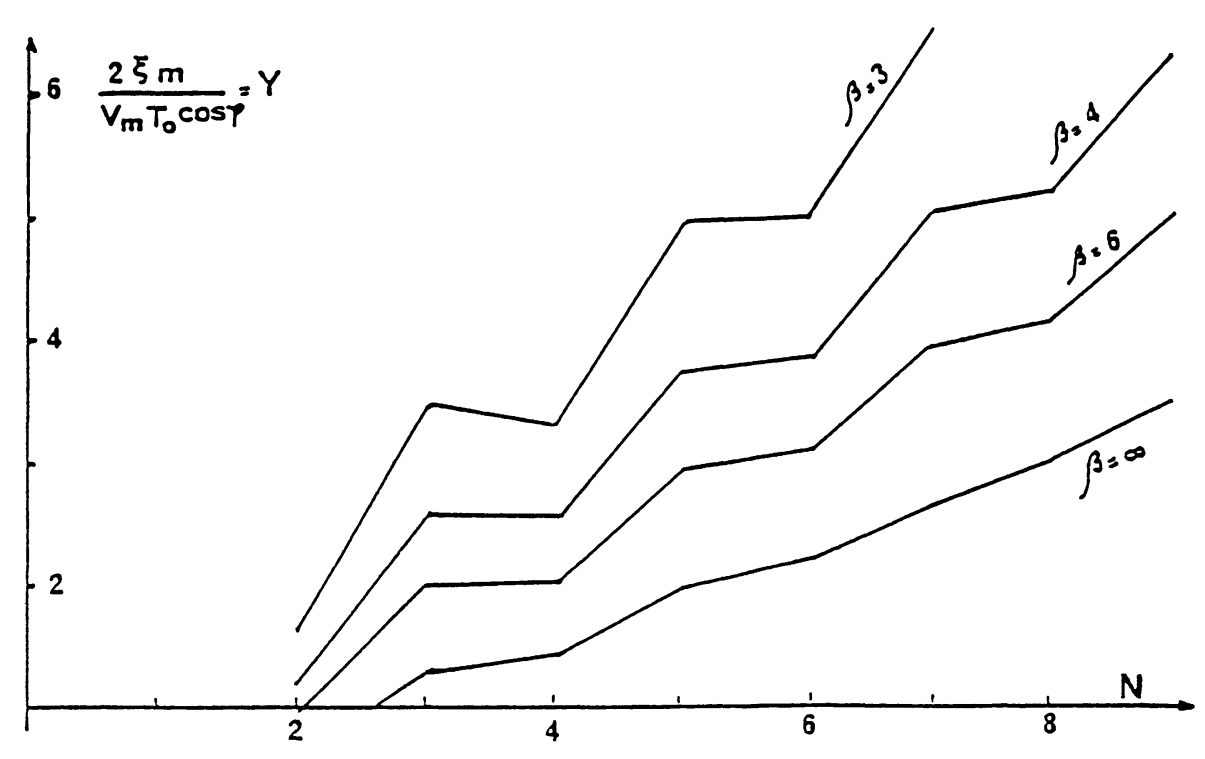

Fig. 6. - Taux de flux total d'induction magnétique admissible en fonction du nombre de séquences $N$ et du gain en courant $\beta\left(\Phi_{N}=(\pi / 2)-\Phi_{0}\right)$.

[Magnetic flux vs. number of steps.]
Pour préciser le flux maximal admissible nous devrons tenir compte des fortes surtensions qui apparaissent au niveau des interrupteurs, et plus particulièrement du premier, au cours des séquences successives. C'est effectivement au cours de la dernière ou avant-dernière séquence (nombre de séquences pair ou impair) que celui-ci est, sur ce point, le plus sollicité.

Dans cette optique, nous mettons en relief le rapport :

$$
Y=\frac{2 \xi_{M}}{V_{M} T_{0} \cos \varphi}
$$

qui met en jeu le flux d'induction magnétique maximal $\xi_{M}$ et la surtension $v_{M}$ admissible par le premier interrupteur. Ce rapport est fonction du gain en courant $(\beta)$ souhaité et du nombre de des spires au sein de la bobine de stockage.

Prenons comme référence la séquence $k$. L'approche de la surtension $V_{p}^{k}$ qui apparaît aux bornes de l'interrupteur $p$ dépend du couplage $M_{p}^{k}$ (et $M_{p}^{k+1}$ ) qui intervient entre les inductances délimitées par les interrupteurs $p$ et $k$ et le reste de la bobine en activité $\ell_{k}\left(\right.$ et $\left.L_{k+1}\right)$. Ainsi nous avons :

$$
\begin{aligned}
V_{p}^{k}=-M_{p}^{k} & \frac{\mathrm{d} j_{k}}{\mathrm{~d} t}-M_{p}^{k+1} \frac{\mathrm{d} i_{k+1}}{\mathrm{~d} t}+ \\
& +\frac{n_{k}-(-1)^{k-p} n_{p}}{n_{k+1}} U_{k} \quad(p<k) .
\end{aligned}
$$

Nous ne présentons ici que la relation afférente aux interrupteurs en situation de recouvrement diélectrique. séquences envisagé. Il dépend de l'état de couplage 
Pour des taux de couplage élevés, au sein de la bobine de stockage, la plus forte surtension apparaît au niveau du premier lors de la dernière $\left(V_{0}^{N-1}\right)$ ou avant dernière séquence $\left(V_{0}^{N-2}\right)$ et nous devrons avoir :

$$
V_{0}^{N-1}<\bigcup_{\mathrm{M}} \quad \text { ou } \quad V_{0}^{N-2}<\vartheta_{\mathrm{M}} .
$$

\section{- Couplage parfait.}

Nous nous plaçons ici (Fig. 6) dans la situation d'efficacité de transfert maximale $\left(\Phi_{0}=\right.$ $\left.(\pi / 2)-\Phi_{N} \cdot\right)$

\section{- Couplage quelconque.}

Cette situation est d'un abord délicat. Cependant moyennant l'hypothèse simplificatrice que le taux de couplage $\left(C_{k}\right)$ entre les inductances élémentaires est indépendant de la séquence considérée $\left(C_{k}=C_{0}\right)$ il est possible d'évaluer la limite par excès de ce rapport (Fig. 7). Le taux de couplage ne peut être trop réduit $\left(C_{0}>0,5\right)$ sous peine de remise en cause de l'instant et du lieu d'apparition de la plus forte surtension.

\section{- Remarque.}

Nous avons supposé que c'est la tenue de l'interrupteur à la surtension crête maximale apparaissant à ses bornes qui s'avérait la plus forte contrainte. Toutefois sa vitesse de recouvrement diélectrique postconduction peut nous imposer des limites plus restrictives. Autrement dit les caractéristiques intrinsèques des interrupteurs utilisés sont déterminantes dans le choix des niveaux d'énergie et des temps de transfert envisageables.

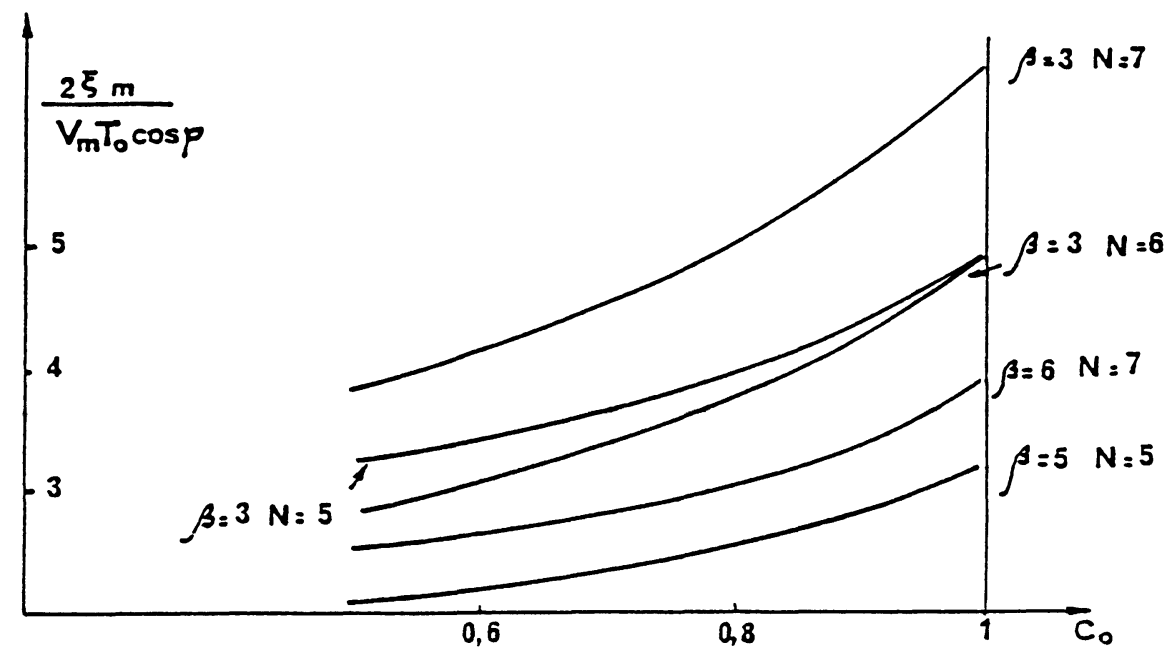

a)

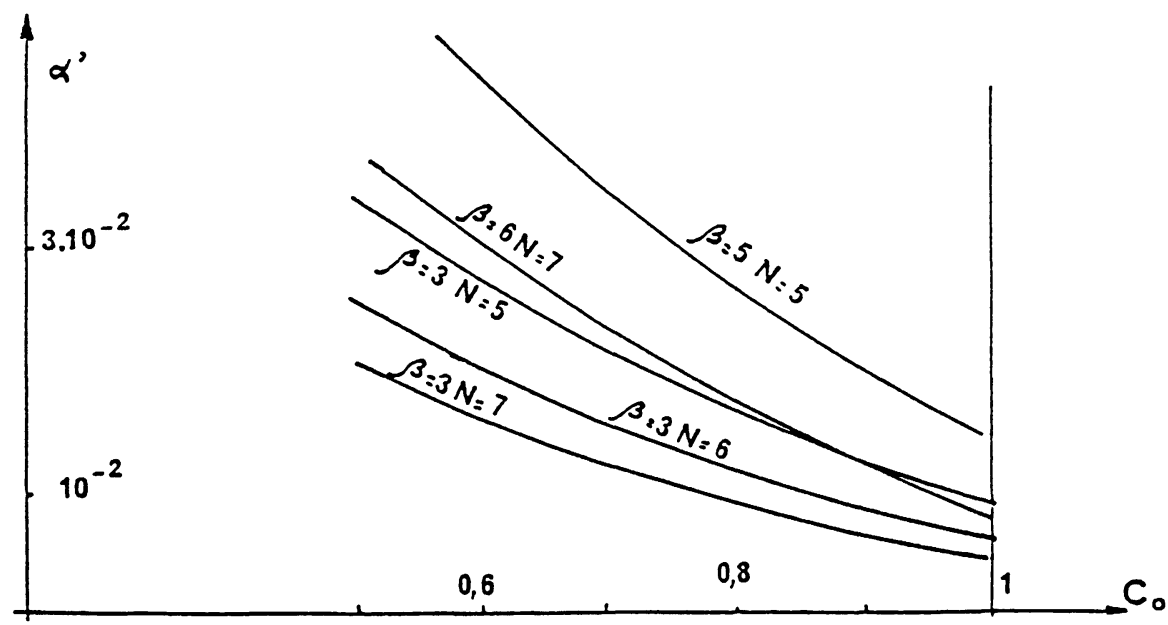

b)

Fig. 7. - Influence du taux de couplage $\left(C_{0}\right)$ sur : a) les taux de flux d'induction magnétique et b) le taux apparent d'énergie de commutation $\alpha^{\prime}$.

[a) Magnetic flux factor as a function of coupling factor. b) Commutation energy factor as a function of coupling factor.] 


\section{Le transformateur.}

Cet élément de base n'apporte aucune difficulté majeure dans l'élaboration du système. Les énergies qui transitent par la capacité sont réduites $\left(\alpha W_{0 T}\right)$, et les puissances correspondantes rapportées à celles des interrupteurs apparaissent minimes.

Quelques précisions peuvent être apportées quant à sa « taille ».

\section{- Section de la culasse magnétique.}

Le flux du champ d'induction magnétique $\psi_{0}$ impliqué dans sa structure magnétique est directement relié au flux des champs d'induction magnétique $\xi_{k}$ mis en cause dans les inductances $L_{0}^{\prime}$ et $L_{k}$. La relation (17) nous permet d'écrire directement :

$$
\psi_{0}=\frac{\xi_{k}}{n_{k}} \alpha \cos \varphi=\frac{\xi_{0}}{n_{0}} \alpha \cos \varphi
$$

(L'état 0 est attribué à la première séquence).

Compte tenu de l'ordre de grandeur des termes $\alpha\left(10^{-2}, 10^{-3}\right)$ et $n_{0}(\approx 50)$ les sections respectives des éléments (bobine de stockage, transformateur), sont dans un rapport excédant aisément 100, même en usant de bobines de stockage à champ magnétique intense (20 à 30 teslas).

\section{- Volume de cuivre du bobinage.}

L'énergie dissipée $\left(\Delta W_{0 T}\right)$ au cours du transfert, dans les bobinages primaires du transformateur, peut être aisément formulée en s'appuyant sur l'hypothèse défavorable que les résistances $\left(R_{k}\right)$ des bobinages sont proportionnelles au nombre de spires $\left(n_{k}\right)$. Lors d'un fonctionnement optimal (couplage parfait) et pleinement efficace $\left(\Phi_{0}=\right.$ $\left.(\pi / 2)-\Phi_{N}\right)$, nous avons

$$
\Delta W_{0 T}=\sum_{k} \int_{0}^{\frac{T_{0}}{2}} R_{k} i_{k}^{2} \mathrm{~d} t_{k} .
$$

Si nous comparons cette perte à l'énergie magnétique initiale admissible, nous obtenons :

$$
\begin{gathered}
\eta=\frac{\Delta W_{0 T}}{W_{0 T}}=\frac{N T_{0} R_{0} I_{0}}{2 \xi_{\mathrm{M}}} \Theta(N, \beta) \approx \frac{N T_{0} R_{0} I_{0}}{2 \xi_{\mathrm{M}}} \beta . \\
\left(2 W_{0 T}=\xi_{\mathrm{M}} I_{0}\right) .
\end{gathered}
$$

Par souci de minimiser les pertes d'énergie et les inductances de fuites nous nous limitons à l'utilisation des seuls bobinages primaires (la capacité est connectée aux extrémités des $n_{0}+n_{1}$ spires). Les pertes formulées représentent alors toutes les pertes par conduction dans le transformateur.

Pour faire apparaître le volume $V_{\mathrm{CU}}$ de conducteur (de résistivité $\rho$ ) nécessaire, nous allons lier la longueur moyenne d'une spire à la section $\left(S_{\mathrm{F}}\right)$ de la culasse magnétique (champ maximal $B_{\mathrm{F}}$ ) :

$$
\langle\ell\rangle=4 k \sqrt{S_{\mathrm{F}}}
$$

et faire intervenir le nombre total de spires $n=n_{0}+n_{1}$. Compte tenu de (21) et de (23) nous obtenons :

$\frac{V_{\mathrm{CU}}}{W_{0 T}} \approx 2 \pi^{2} k^{2} \frac{\rho}{\eta B_{\mathrm{F}}} \frac{n}{N} \frac{\beta}{v_{\mathrm{M}} Y}\left(1-\frac{4}{\pi \beta}\right)^{2}$

( $Y$ résulte du calcul, cf. Figs. 6 et 7 ).

En nous référant à des valeurs raisonnables des grandeurs impliquées, ce rapport atteint quelque $10^{-3} \mathrm{~m}^{3} / \mathrm{kJ}$ pour $1 \mathrm{kV}$ de surtension admissible $\left(\beta=5 ; \rho=2 \times 10^{-8} ; \quad B_{\mathrm{F}} \approx 1 \mathrm{~T} ; \quad n / N \approx 10 ;\right.$ $\left.\eta=5 \times 10^{-2}\right)$.

\section{Conclusion.}

Nous venons de préciser le comportement d'un système original de transfert d'énergie à haut rendement entre deux inductances. Nous avons pu appréhender l'évolution des différentes grandeurs électriques et montrer que ce fonctionnement séquentiel, qui fait appel à un bloc de condensateurs, exige une faible énergie capacitive installée (quelque $10^{-3}$ fois l'énergie magnétique initiale) et un transformateur de faible puissance. Nous avons donc là, une solution potentielle aux problèmes de transfert de hautes énergies dans les systèmes impulsionnels.

Cette étude met toutefois en relief le rôle crucial des interrupteurs. Le flux d'induction magnétique et, par voie de conséquence, l'énergie admissible sont tributaires de leurs performances. Les conjoncteurs disjoncteurs à vide, étudiés par ailleurs, paraissent être ici, un moyen de commutation acceptable [8] ; ils permettent d'envisager d'ores et déjà (puissance de commutation $10^{9}$ VA) des transferts d'énergie à des niveaux de l'ordre de $100 \mathrm{~kJ}$.

Bibliographie

[1] Rıoux C., Théorie simplifiée des procédés de transfert inductif à haut rendement, Revue Phys. Appl. 10 (1975) 75-79.
[2] Legentil M., Rioux C., Caractéristiques des transferts inductifs à haut rendement par procédé dissipatif, Revue Phys. Appl. 11 (1976) 337-342. 
[3] Giorgi D., Lindner K., Long J., Navapanich T., ZUCKER O., Proof of principle experiment of meatgrinder: an inductive energy storage and transfert circuit, Proceeding of $\mathrm{V}^{\text {th }}$ Pulsed power conference (Arlington, juin 1985) p. 295.

[4] Giorgi D., Lindner K., Long J., Navapanich T., ZUCKER O., The design and analysis of multi stage meatgrinder circuit, Proceeding of $\mathrm{V}^{\text {th }}$ Pulsed power conference (Arlington, juin 1985) p. 615.

[5] Delvaux J., Etude de la surtension de réisolement d'interrupteurs à explosion de feuille pour des courants très intenses, Université d'Orsay, Thèse de $3^{\mathrm{e}}$ cycle (septembre 1975).
[6] VitKovitsky M., SCHERRER V., Recovery characteristic of exploding wire fuses in air and vacuum, J. Appl. Phys. 52 (1981) 3012-3015.

[7] Allano S., Delmas A., Rioux C., Study of a non dissipative transfer system and tests on related vacuum switches, Proceeding of VI $^{\text {th }}$ Pulsed power conference (Arlington, juin 1987) p. 449.

[8] Bauville G., Delmas A., Haddad N., Rioux C., Study of recovery phenomena in high current pulse triggered vacuum switch, $13^{\mathrm{e}}$ Symposium International sur les Décharges et l'Isolement Electrique dans le Vide (Paris, 1988) p. 404.

[9] WeLdon W. F., Pulsed power packs a punch, IEEE Spectrum, 59-66 March 85. 